\title{
Pérdidas a lo largo del ciclo vital en adultos mayores
}

\section{Losses throughout the life span in old age people}

\author{
Karen Cecilia Sandoval Carlos, María Dolores García Sánchez \\ y Oliva Eréndira Luis Delgado ${ }^{I}$
}

Citación: Sandoval-Carlos, K.C., García-Sánchez, M.D. y Luis-Delgado, O.E. (2019). Pérdidas a lo largo del ciclo vital en adultos mayores. Psicología y Salud, 29(1), 79-90.

\section{RESUMEN}

Con el aumento de la población de personas mayores en las últimas décadas, resulta pertinente suponer que aumentarán igualmente sus necesidades emocionales, físicas y económicas, por lo que es importante ahondar más en los aspectos psicológicos del envejecimiento y en las vivencias de mayor significado en esa etapa. Por lo anterior, los objetivos de este estudio fueron los de conocer en un grupo de adultos mayores sus pérdidas más importantes en cada una de las etapas del desarrollo, y analizar las posibles diferencias de género en la percepción de dichas pérdidas. Para ello, se realizó una investigación cualitativa con veintiséis participantes, a quienes se hicieron entrevistas semiestructuradas. Se hizo un análisis de contenido de las mismas, teniendo como categorías principales las pérdidas humanas, de salud, del trabajo, materiales o de habilidades. Los resultados mostraron que para los adultos mayores las pérdidas más dolorosas a lo largo de su vida fueron el fallecimiento de padres, hijos y parejas, hallándose claras diferencias respecto al género. En conclusión, puede decirse que las pérdidas humanas tuvieron un mayor impacto que las de otro tipo, y que especialmente la muerte de un hijo resulta ser uno de los duelos más complicados.

Palabras claves: Adultos mayores; Pérdidas; Ciclo vital; Duelo.

\begin{abstract}
The increase in the population of elderly people in the last decades, it is only natural to expect that their emotional, physical and economic needs will also increase, thus making it important to further explore the psychological aspects of aging and its most significant experiences. The present study sought to identify elderly adults' most important losses at different stages of development. Another objective consisted of exploring gender differences in the perception of such losses. A qualitative, cross-sectional study with 26 elderly participants was conducted. Semi-structured interviews and content analysis led to the main findings. Analysis categories included the loss of human, health, work, material, and personal abilities. Results showed that the most painful losses throughout their whole lives were the death of the parents, children or spouse; with clear differences according to gender. In conclusion, it is proposed that human loss had a greater impact than other kinds of loss, and that the death of one's own children leads to one of the most complicated bereavements.
\end{abstract}

Key words: Older adults; Losses; Life span; Bereavement.

\footnotetext{
${ }^{1}$ Universidad Autónoma de Zacatecas, Av. Preparatoria s/n, Col. Progreso, 98000 Zacatecas, Zac., México, tel. (467)924-19-34, correos elec-
} trónicos: kcecisc@gmail.com, dogasa05@gmail.com y oliva@uaz.edu.mx. Artículo recibido el 11 de febrero y aceptado el 11 de junio de 2018. 


\section{INTRODUCCIÓN}

$\mathrm{D}$ e acuerdo con el Consejo Nacional de Población (2014), la disminución de la mortalidad se traducirá en una mayor esperanza de vida para la población. Las estimaciones para el año 2020 indican que el grupo de 65 años y más representará $8.2 \%$ del total de la población mexicana y $10.2 \%$ para 2030 . Por tal motivo, deben analizarse las necesidades futuras que tendrán esas personas en los aspectos económico, social, emocional y de salud. En este último rubro, debe tenerse en cuenta que es en esta etapa en la que se presenta la mayor prevalencia de uno de los problemas más temidos de la vejez: la dependencia funcional. Es por ello que los retos para las políticas públicas serán, por un lado, la generación de espacios de trabajo para quienes continúen en el mercado laboral con una protección adecuada, así como el aumento del sistema de pensiones.

Además, debe considerarse que los adultos mayores que han experimentado en un corto periodo múltiples pérdidas de cualquier tipo, que no disponen de un apoyo social adecuado y que no han logrado adaptarse satisfactoriamente a los estresores actuales y del pasado, tienen una mayor predisposición para desarrollar trastornos mentales tardíos en la vida, según afirman Cruz, Jara y Rivera (2010), tales como la depresión o ciertos padecimientos psicosomáticos (Rivera y Montero, 2008).

Para lograr una comprensión más amplia de esa etapa, es necesario ir más allá del aspecto puramente biológico, en el cual el envejecimiento refiere a un proceso cronológico de declive, $\mathrm{y}$ ahondar en la parte psicológica en función de los cambios de personalidad y en el ámbito social, que implica la percepción, participación e integración en la sociedad de la persona mayor (Baltes, 1987; Erikson y Erikson, 1997).

El desarrollo humano involucra tanto constancia como cambio en el comportamiento a lo largo de toda la vida, pues en cada una de sus etapas hay ganancias y pérdidas, trátese de la infancia o de la adultez mayor. Esta idea fue la que dio origen a la perspectiva teórica del ciclo vital, que propuso una forma diferente de ver el desarrollo, al que Baltes, Lindenberger y Staudinger (1998) redefinen como un "cambio selectivo, relacionado con la edad, en la capacidad adaptativa" (p. 17).

Se entiende que el desarrollo engloba un equilibrio entre crecimiento y declive, y por tanto en toda etapa la persona puede ganar en un área y perder en otra; en el caso del adulto, capacidades como su vocabulario pueden continuar aumentando, mientras que en otras, como la solución de problemas no familiares, disminuyen. De igual manera, pueden surgir algunas habilidades, como la experiencia en un área determinada. Según esta teoría, ningún cambio en el desarrollo de la vida es únicamente ganancia. Aunque si bien es cierto que la proporción entre ganancias y pérdidas suele variar a lo largo del ciclo vital, en la adultez mayor generalmente ocurren más pérdidas que ganancias.

Es por ello que se plantean tres procesos fundamentales en el desarrollo a lo largo de toda la vida, según Baltes (1987):

El crecimiento: Engloba la ganancia y el alcance de niveles de funcionamiento cada vez más eficaces y complejos que hacen posible a la persona avanzar en las diferentes etapas de la vida, pues cada una depende de los sucesos que ocurrieron en las que la antecedieron.

El mantenimiento: Ante situaciones de riesgo, la persona trata de mantener el funcionamiento actual $\mathrm{o}$, ante una pérdida, regresar a niveles previos de funcionamiento, de manera que pueda encontrarse en un estado óptimo.

La regulación frente a las pérdidas: Frente a una pérdida de recursos internos (como la disminución de la capacidad de memoria) o externos (como la jubilación o la pérdida de un ser querido), la persona trata de reorganizar el funcionamiento para mantenerlo en los niveles habituales. Uno de los mecanismos para lograr la reorganización es la compensación, que Baltes y Baltes (1990) y Baltes y Dickson (2001) explican en su modelo de selección, optimización y compensación diciendo que en la adultez mayor el individuo elegirá y concentrará sus esfuerzos en aquellas actividades que aún puede desempeñar, actuando como un equilibrio ante la pérdida de alguna habilidad.

De tales conceptos se desprende la idea de que a lo largo del desarrollo los recursos y las metas van variando; mientras que durante la infancia 
se pretende alcanzar el mayor crecimiento posible, durante la vejez el mantenimiento y la regulación ante las pérdidas se convierten en la prioridad, por lo que la persona dedica la mayoría de los recursos disponibles a esas tareas (Villar, 2005).

Al referirse a una pérdida, la mayoría de las veces se piensa en la muerte de un ser querido; sin embargo, como afirma Tizón (2004), el dejar de tener ciertas capacidades o habilidades personales, bienes patrimoniales, amistades u otras situaciones pueden también considerarse como pérdidas. A su vez, Neimeyer (2007) explica que siempre que la persona deje de tener algo que poseía un valor real o simbólico en cualquier ámbito de su vida, tendrá una pérdida que generará en ella dolor emocional y a la que deberá adaptarse.

Respecto a los estudios realizados sobre las pérdidas, la mayoría de las investigaciones sobre los adultos mayores se han enfocado principalmente en conocer el impacto que tiene en ellos la muerte de la pareja (Caserta, Utz, Lund, Swenson y De Vries, 2014; Damianakis y Marziali, 2012; Holland, Futterman, Thompson, Moran y Gallagher-Thompson, 2013), el dolor producido por la pérdida de un hijo (Lichtenthal, Currier, Neimeyer y Keese, 2010; Liu et al., 2015) y cómo se vivencia el proceso del envejecimiento (Bagheri-Nesami, Rafii y Oskouie, 2010; Dockendorff, 2014; Krzemien, Urquijo y Monchietti, 2008; Stefani, 2004).

Particularmente en México, destaca la propuesta de Rivera y Montero (2008) en la que se explican las pérdidas experimentadas por los adultos mayores, argumentando a la par que Baltes et al. (1998) que en toda etapa de la vida se presentan ciertos cambios que engloban tanto pérdidas como ganancias, pero que en esta fase del ciclo vital en especial los recursos de la persona se orientan a la recuperación ante las pérdidas. En el instrumento que dichos autores elaboraron se categorizan las pérdidas en cinco áreas: a) El cuerpo. Dificultades en la funcionalidad física (disminución de la fuerza o el vigor). b) Las relaciones objetales (sociales). Todos los vínculos que ya no se tienen en la realidad o en la fantasía y que no hay posibilidad de reemplazar. c) El ser para el mundo. Los roles derivados del trabajo que permiten a la persona sentirse útil y productiva para la sociedad y que se pierden con la jubilación. d) El ser en el mundo.
Como resultado de la misma pérdida del "ser para el mundo", el contexto actual comienza a perder significado, pues la sociedad actual se vuelve cada vez más distinta de la que conoció en su pasado debido al avance tecnológico, por lo que la persona se siente en ocasiones "fuera de época". e) $L a$ identidad. A consecuencia de las pérdidas anteriores, la persona se percata de que tiene que reestructurar la meta o el ideal de sí misma que quería alcanzar; es por ello que a veces le resulta más sencillo refugiarse en la persona que fue y en sus logros del pasado.

Debido a la escasa investigación acerca de tales pérdidas desde la perspectiva del ciclo vital, resulta necesario indagar cómo han ocurrido en cada una de las citadas etapas para comprender mejor las que se experimentan en la adultez mayor.

En la presente investigación cualitativa de corte transversal, cuyos objetivos fueron conocer en un grupo de adultos mayores las pérdidas más importantes para ellos en cada una de las fases del desarrollo y analizar si había diferencias de género en la percepción de dichas pérdidas, se tomaron como referentes los postulados teóricos del ciclo vital de Baltes (1987) y Baltes et al. (1998), así como la "estructura de pérdidas" de Rivera y Montero (2008), pues permiten tener una perspectiva más amplia de las vivencias de los adultos mayores, en especial para entender las pérdidas como una parte necesaria del desarrollo que aparece conjunta e inseparablemente con las adquisiciones o ganancias en cualquier edad.

\section{MÉTODO}

\section{Participantes}

Los participantes fueron veintiséis adultos mayores: trece hombres y trece mujeres, residentes en el estado de Zacatecas (México), cuyas edades fluctuaron entre 60 y 85 años en el caso de las mujeres, y entre 60 y 73 años en el de los hombres. Algunas de las participantes (34.6\% del total) asistían a un club social del Sistema Nacional para el Desarrollo Integral de la Familia (DIF), ubicado en una colonia de nivel socioeconómico bajo, y los demás participantes $(65.4 \%$ del total $)$ residían 
en una colonia de nivel socioeconómico medio. La mayoría de los participantes estaban casados y vivían con su pareja.

Los principales problemas de salud referidos por los entrevistados fueron, a saber: problemas de audición y visión (30.8\%), dolores articulares $(26.9 \%)$ y diabetes (19.2\%); más la mitad de los participantes manifestaron tener más de una enfermedad. En el caso de tres de ellos (11.54\%), sus padecimientos constituían graves limitantes para el desempeño de sus actividades cotidianas, e incluso dos se retiraron de su trabajo debido a sus enfermedades (dolor de columna vertebral y problemas pulmonares).

En el aspecto mental, todos los participantes estaban en pleno uso de sus facultades; la mayoría manifestaba una ubicación adecuada en tiempo, espacio y persona, y sólo algunos de ellos se confundieron en ocasiones o no recordaban la fecha, pero en la narración de hechos autobiográficos en general no tuvieron problemas para recuperar la información solicitada. Su discurso fue fluido y coherente durante las entrevistas y mostraron una adecuada atención. A uno de los participantes ciertas preguntas tuvieron que repetírsele debido a un problema de audición. Todas las personas mostraban una movilidad adecuada que les permitía desplazarse por sí mismos sin ayuda alguna.

\section{Recopilación de datos}

Para recolectar la información, se hicieron a los participantes entrevistas semiestructuradas, teniendo como referente una guía de preguntas que fueron agrupadas de acuerdo a las cinco etapas del ciclo vital: infancia, adolescencia, juventud, adultez y adultez mayor, en cada una de las cuales se indagaron los diversos tipos de pérdidas vividas (véase Anexo).

Las entrevistas se elaboraron con base en los postulados teóricos del ciclo vital de Baltes (1987) y Baltes et al. (1998), quienes indican que para comprender de manera más integral las pérdidas que vivencia el adulto mayor, debe tenerse en cuenta la forma en que ha experimentado y sobrellevado otras pérdidas en las etapas anteriores de su vida. Esas pérdidas se categorizaron en función de la clasificación de Tizón (2004), que habla de pérdidas intrapersonales (aspectos o partes del yo, como funciones cognitivas y profesionales), relacionales (muerte o separación de personas significativas), materiales (posesiones) y evolutivas (ligadas al desarrollo); de manera específica, para profundizar en la etapa de la adultez mayor se tomó en cuenta la propuesta de estructura de pérdidas de Rivera y Montero (2008).

\section{Procedimiento}

Se contactó y pidió permiso para asistir al citado club social del DIF. Una vez otorgado, una de las investigadoras fue observadora participante por aproximadamente ocho meses. Hechas las observaciones, se les explicó la finalidad principal del estudio, que consistía en conocer las vivencias de las personas mayores. Quienes accedieron a participar firmaron una carta de consentimiento informado, aceptando que las entrevistas fueran grabadas.

La duración de las entrevistas varió, ya que en el caso de las mujeres adultas mayores que asistían al club social se realizaron por la tarde, en el horario en el que normalmente asistían, y tuvieron una duración mínima aproximada de dos horas y una máxima de tres horas por sesión. Los espacios fueron las mismas instalaciones. El hecho de que la mayoría acudiera diariamente al club facilitó que las entrevistas pudieran extenderse hasta dos o tres sesiones, resultando por tanto entrevistas con una duración total de seis horas en promedio por persona.

Con quienes no acudían al club social se concertaron citas previamente para designar un espacio durante la tarde. Por lo general, las entrevistas se hicieron en los mismos hogares, si bien en estos casos, por cuestiones de tiempo, en una sola sesión, aunque extendiéndose de dos a tres horas por persona.

Luego se llevó a cabo un análisis de contenido de las entrevistas, para lo cual, según las perspectivas teóricas de Tizón (2004) y de Rivera y Montero (2008), se identificaron los principales indicadores y las dimensiones relevantes para el presente estudio, distribuyendo las respuestas obtenidas en las siguientes categorías: infancia robada, pérdida de un ser querido (por muerte, separación física, emocional o divorcio), del cuerpo (como la salud o fuerza física), de la libertad (debido 
al matrimonio) y pérdida por la jubilación y de “el-ser-en-el-mundo"(época actual distinta a la de su infancia). Se utilizó el programa Atlas-Ti para vaciar los datos de las entrevistas y organizar las categorías ya mencionadas.

\section{RESULTADOS}

Los resultados que se muestran a continuación son extractos de las respuestas de los participantes. Por cuestiones de espacio, sólo se eligieron algunas respuestas que ejemplifican los hallazgos; cabe señalar que los nombres de los participantes que aparecen son ficticios debido al acuerdo de confidencialidad y para mantener encubierta su identidad.

Durante la infancia y la adolescencia, tanto hombres como mujeres experimentaron la llamada "infancia robada", esto es, el abandono de los roles infantiles, como jugar y estudiar, por tener que trabajar debido a la precaria situación económica de sus familias, si bien fue hasta la adolescencia cuando los entrevistados parecen haber tenido una mayor conciencia de lo que significaba haber perdido la oportunidad de continuar sus estudios:

"Yo digo que me maduraron muy pronto, porque ya cuando salí de la primaria a mí ya no me consideraban un niño; me trataban como un adulto porque ya trabajaba. Y yo digo que me abandonaron, porque tratar a un niño como un adulto era muy feo. Mi mamá me dijo que tenía que cooperar en la casa. Ya no me compraron ropa ni juguetes porque se consideraba que ya era un hombre, pero yo no me sentía un hombre" (Jaime, 68 años).

Los participantes que debieron trabajar en su infancia refieren que la responsabilidad asignada fue demasiada para una etapa tan temprana, y debido a ello en algunas ocasiones llegaron a sentirse abrumados y solos, pues lo esperado es lograr la independencia económica, pero no durante la infancia sino hasta la juventud, cuando ya se tienen mayores recursos para convertirse en una persona autónoma (Lefrançois, 2001).

Las pérdidas más dolorosas en esas etapas del desarrollo fueron la del padre (por muerte, separación o divorcio), el fallecimiento de los abuelos, que para muchos fueron quienes más los cuidaron, y de familiares y amigos.
Para uno de los participantes varones, no haber contado con su padre durante su infancia y casi toda su adolescencia lo afectó durante toda su vida, pues consideraba que creció solo, y que de haber conocido antes a su padre y haber vivido con él habría tenido apoyo suficiente para continuar sus estudios:

"Mi infancia fue muy triste porque primeramente mis padres son divorciados [...] Los [años] que tengo ya, 72, son los que estuvieron separados. Mi papá no vivió conmigo y mi jefecita se me fue hace cuatro años. Al no contar uno con su padre, no tiene el apoyo de nadien [sic]. Tiene uno que sobrevivir de la forma que sea, hay que subir pa' [sic] arriba y no ir para abajo, porque se ladea uno, y como un árbol se va uno de lado y se cae. Cuando uno no tiene ese apoyo, pues tiene que valerse por sí mismo" (Fernando, 72 años).

Sin embargo, para otros participantes el fallecimiento del padre sucedió a una edad tan temprana que no asimilaron entonces la trascendencia de esa pérdida, pues varios de ellos no comprendían aún el concepto de la muerte.

Una de las mujeres refirió que haber dejado de ser hija única fue un suceso complicado, pues a la llegada de sus hermanos varios años después de haber nacido ella no solo implicó compartir el amor y la atención de sus padres, sino que se le asignó también el rol de madre-cuidadora.

Para las mujeres, haber experimentado en su juventud la muerte de algunos de sus hijos aún bebés, de sus madres y de otros familiares fue uno de los eventos más dolorosos. Ante la pregunta hecha a Rosita, de 78 años, de cuántos hijos tiene, responde:

"Vivos, once, pero tuve dieciséis. Los otros se murieron chiquitos. Los últimos que tuve fueron gemelos [...] Como los primeros que perdí estaban chiquitos, pues sí me daba tristeza porque no es lo mismo que ya los veía ahí, que los bañaba, y a los tres meses ya no los tenía”.

Oviedo, Urdaneta, Parra y Marquina (2009) explican que cuando un hijo nace muerto o muere alrededor del nacimiento, la madre se enfrenta a un doble duelo: la pérdida del hijo y la frustración de su maternidad, proceso que puede ser incluso más severo que el deceso de un adulto. En el caso de Rosita, sin embargo, perder a sus hijos a muy 
temprana edad no fue tan difícil, según refiere. Para ella, un suceso más complicado fue la muerte de su esposo.

Durante su juventud, algunas de las participantes experimentaron la viudez, la que afrontaron como uno de los momentos más difíciles de su vida al sentirse desestabilizadas emocional y económicamente para mantener a sus hijos. Entre los varones se presentó un circunstancia singular, pues explicaron que, al casarse, una de las cosas que perdieron fue su libertad; es decir, ante las nuevas responsabilidades adquiridas al tener una familia, debieron reorganizar sus rutinas y dejar atrás ciertos intereses y pasatiempos que eran de su agrado y que formaban parte de su vida cotidiana.

"Y dejé el mundo mundano para dedicarme a la familia, y tardé un tiempo en acostumbrarme a dejar el mundo de los placeres por el de los quehaceres, y no está fácil [...] Me entretuve unos años en aceptar que ya no estaba soltero, que ya había perdido mi libertad, porque ya se hace uno codependiente de la pareja. No puedo ir solo sino que vamos en pareja" (Diego, 60 años).

Para este participante fue complicado adaptarse a su nuevo estilo de vida, porque antes de casarse no tenía responsabilidades hogareñas, pero una vez que contrajo matrimonio a los 29 años de edad tuvo que adecuarse a nuevos deberes y labores e incluir a su esposa en sus actividades. Como apunta Erikson (1997) en su teoría del desarrollo psicosocial, en esta etapa surge un fuerte deseo de compartir la vida con alguien más en un compromiso duradero, aun cuando eso involucre sacrificios significativos.

Luego, en la adultez, las mujeres reportaron haber perdido a sus padres; para algunas, la muerte de la madre fue una de las más significativas en su vida. Otras también dijeron haber perdido a su esposo al divorciarse, así como a sus hijos. En el caso de las hijas adultas que se convirtieron en las cuidadoras principales de sus padres, fue más difícil adaptarse a la muerte de los mismos.

Ya como adultos, los hombres señalaron que las muertes más impactantes fueron la de los padres y la abuela. En otro aspecto, uno de los entrevistados dijo que a causa de una inadecuada intervención quirúrgica perdió casi toda la visión de uno de sus ojos, por lo que tuvo que dejar de conducir vehículos.
Para las mujeres, la pérdida de la pareja por muerte o por divorcio fue de los eventos más complicados de su vida:

"Me divorcié cuando tenía -según me parece- cincuenta y dos, y a raíz de eso mi familia se dividió. Mis hijos tomaron partido y haga de cuenta que se hicieron dos bandos. Tomaron rivalidad con todo lo que se dice, la alevosía y ventaja [...] Al final de la vida, no era una mujer, era un hombre por el que me dejó. Entonces él se sentía terriblemente mal. A mí me quería para que yo estuviera así, ante la gente, fingiendo aquel matrimonio. A mí nada más me quería para aparentar y [poder] llevar él su doble vida". Esta participante se arrepentía de haberse permanecido viviendo en la misma casa durante un tiempo: "Sí, de haberme quedado, pero ya qué, el daño ya está hecho [1lora] y es terrible... porque me siento terriblemente mal" (Dolores, 67 años).

Esta misma participante dijo que su esposo la había agredido mucho cuando se divorciaron, porque si bien vivían en la misma casa, cada quien lo hacía en su respectiva mitad; además indicó que le dolía mucho que aquél hubiera "envenenado" a sus hijos diciéndoles cosas sobre ella que no eran ciertas, que los manipulara y que no le hablaran, porque ella esperaba que aunque su matrimonio terminara, su familia seguiría unida, pero sus hijos se separaron de ella y varios de ellos aún no le hablaban. Esto es explicado por Oramas (2014) en términos de que el divorcio es un evento de transición estresante que involucra crisis emocional, cambios en el comportamiento y, en general, caos o desajuste en la vida.

Respecto a la muerte de la pareja hubo diferencias de género, toda vez que más de la mitad de las mujeres eran viudas y sólo uno de los varones entrevistados, lo cual concuerda con resultados previos de Montes y Macedo (2013). Las mujeres han descrito ese suceso como uno de los eventos más desestructurantes (Bellamy, Gott, Waterwoth, McLean y Kerse, 2014; Damianakis y Marziali, 2012; Holland et al., 2013). El hecho de que la muerte de la pareja tenga un mayor peso emocional para la población femenina puede deberse a los estereotipos culturales que definen casi por completo la identidad de una mujer en función de sus roles de madre y esposa, y por tanto, al morir el varón, no sólo pierden a un ser querido sino 
también una parte importante de su yo (Montes y Macedo, 2013).

Los participantes expresaron que en la etapa de la adultez mayor las principales pérdidas que habían experimentado eran sobre su cuerpo, las que se traducían como una disminución de la salud y la fuerza física, así como en sus relaciones sociales, sobre todo por la muerte de los padres y la pérdida del apoyo de su pareja, resultados también encontrados por Rivera y Montero (2008). También refirieron tener al menos un problema físico que incluso en ocasiones se había convertido en un obstáculo para realizar ciertas actividades cotidianas, tal como afirman Dockendorff (2014) y Li, Chi y Xu (2013) (Tabla 1).

Tabla 1. Pérdidas en la adultez mayor.

\begin{tabular}{|c|l|}
\hline GÉNERO & \multicolumn{1}{c|}{ PÉRDIDAS MÁS COMPLICADAS } \\
\hline \multirow{3}{*}{ Hombres } & $\begin{array}{l}\text { • Pérdidas de la capacidad y la salud física. } \\
\text { • Pérdidas humanas: muerte de padres e hijos. } \\
\text { - Jubilación. } \\
\text { • Pérdida de "el-ser-en-el-mundo". }\end{array}$ \\
\hline \multirow{2}{*}{ Mujeres } & $\begin{array}{l}\text { • Pérdidas de la capacidad y la salud física. } \\
\text { - Pérdidas humanas: muerte de padres e hijos y pérdida del apoyo de la pareja. } \\
\text { • Pérdida de "el-ser-en-el-mundo". }\end{array}$ \\
\hline
\end{tabular}

Nota: Las categorías que aquí se incluyen son un esbozo representativo de las pérdidas que los participantes refirieron experimentar únicamente en la etapa de la adultez mayor. No se incluyen las otras fases del desarrollo.

Sobre la jubilación, fue sobre todo en los hombres en quienes tuvo un efecto negativo haber dejado su trabajo; en realidad, pocas de las mujeres entrevistadas trabajaban fuera del hogar:

«Me afectó en mi salud. En el trabajo ya me sentía muy agotado. Veía que me iba quedando atrás, dije "Ya no tiene caso", y aunque me dijeron que me preparara para ese cambio, pues hice caso omiso de lo que me comentaron, pensando que la libertad sería para estar más a gusto, acostado más rato, y nunca fue así». (Andrés, 69 años).

Este participante había trabajado cuarenta y cuatro años como docente, y para él fue muy complicado dejar de laborar siete años atrás. Estaba acostumbrado al ritmo, la actividad y el horario. Señaló que para él andar sin reloj era como andar desnudo, porque como maestro siempre tenía que estar pendiente de la hora. De cierta manera había compensado el ya no ir a dar clases asistiendo todavía los lunes a honores a la bandera en alguna de las diferentes escuelas en las que laboró. También asistía a un grupo político en el que hablaban de temas actuales, y todavía los domingos se ponía a leer como si estuviera preparando el material para sus clases. En cierta forma, eso denota que aún no dejaba del todo su trabajo, pues seguía llevando a cabo actividades de cuando era profesor. Para él, la jubilación había incidido principalmente en sus problemas de salud, pues sufría dolores de columna, y ahora que su actividad física se había reducido, resentía más ese padecimiento.

Asimismo, en la adultez mayor ocurre la pérdida de "el-ser-en-el-mundo". Se halló similitud con los hallazgos de Rivera y Montero (2008), pues varias de las personas mayores entrevistadas dijeron que las épocas en la que vivieron su juventud y la actual eran muy distintas, principalmente en la práctica de ciertos valores y en la educación que les inculcaron.

Uno de los varones entrevistados dijo que a causa de una mala intervención quirúrgica casi había perdido la visión de uno de sus ojos, por lo que tuvo que adaptarse y dejar de manejar. Baltes y Baltes (1990) y Baltes y Dickson (2001) aluden a un proceso de compensación por el que, ante una pérdida o ausencia de algún recurso importante para lograr lo que se quiere, se adquieren o se reconstruyen nuevos medios para sustituir los que ya no están disponibles o que se han perdido; en el caso de este participante, había optado por utilizar otros medios de transporte al ya no poder conducir.

Uno de los resultados inesperados en esta fase del ciclo vital fue la insatisfacción marital de algunas de las mujeres participantes, manifestada sobre todo en una comunicación inadecuada, escasa 
expresión de afecto y ausencia del apoyo de su pareja en su vida cotidiana:

«Con el cáncer, al principio sí estuvo mi esposo conmigo. Ya después de las quimios, en los controles, él ya no se interesó por saber qué me pasaba [...] La relación se va viendo diferente y se cambia por completo [...] Tú tienes tu pareja y tú dices "Él es el que debe estar conmigo; sabe que voy y sabe que vengo y no me pregunta a qué voy o que me dijeron". Y tú dices: "Yo voy a vivir para mí y no me importa que no me apoye el que no debe de apoyarme", porque uno siente que ni siquiera lo debe de pedir, y pues adelante: "Yo, yo"» (Nancy, 62 años).

Dicha participante había padecido cáncer y comentó que había sido un momento difícil en su vida, porque al principio pensaba que iba a morir. Durante el tratamiento, las personas que más la apoyaron fueron su esposo y una hermana, pero cuando pasó del tratamiento a las revisiones, aquél dejó de apoyarla; no la acompañaba ni le preguntaba acerca de ello. En una ocasión que lo confrontó le respondió que así era su forma de ser y que no iba a cambiar, de manera que ella asimiló paulatinamente el hecho de que aunque deseara el apoyo de su marido, en realidad ya no lo tenía, y por tanto se concentró en sí misma, en mostrar fortaleza y en superar el problema por sus propios medios.

Estos resultados se contraponen a los hallazgos de Villar, Villamizar y López (2005) y Arias y Pollizi (2011), quienes expresan que la satisfacción marital suele aumentar durante la adultez mayor debido a factores tales como una mejor comunicación, la superación de ciertas dificultades y un apoyo emocional constante.

\section{DISCUSIÓN}

Be, Whisman y Uebelacker (2013) explican que las diferencias de género en la percepción de la satisfacción marital podrían deberse a que a la mujer se le forma socialmente para ser más sensible y atenta a la calidad de sus relaciones interpersonales. Por otra parte, la experiencia compartida por algunos padres que habían perdido a un hijo parece ser un hecho con el que seguían lidiando emocionalmente. Varios autores coinciden en que la muerte de un hijo constituye uno de los eventos más estresantes de la vida, misma que puede traducirse en un duelo más riguroso e intenso cuando se le compara con el que generan otras pérdidas humanas (Endo, Yonemoto y Yamada, 2015; Rando, 1985), y que incluso puede perdurar a través de toda la vida de los padres (Lichtenthal et al., 2010).

En conclusión, en el presente estudio se observó que las pérdidas de mayor impacto en $62 \%$ de las mujeres y 54\% de los hombres participantes fueron principalmente la muerte de los progenitores durante la juventud, la adultez y la adultez mayor, así como el fallecimiento de un hijo en cualquier etapa de la vida (vivenciado por $27 \%$ de la población total). Se encontraron diferencias de género en el caso de la viudez y la pérdida del apoyo de la pareja, mismas que fueron pérdidas con mayor efecto en las mujeres, mientras que la jubilación y la pérdida de la libertad debida al matrimonio fueron los sucesos más significativos entre los hombres. No se hallaron diferencias significativas en cuanto al nivel socioeconómico de los participantes. Cabe subrayar que ante las numerosas pérdidas que habían experimentado, la mayoría de ellos mostraba un adecuado proceso de ajuste y adaptación.

El presente estudio tiene aplicaciones prácticas de gran relevancia. Al analizar las principales pérdidas de las personas mayores a lo largo de su vida, pueden comprenderse mejor las que ocurren actualmente para, de esta manera, proporcionar herramientas que hagan posible su mejor manejo y adaptación. Además, la presente investigación puede ser útil como un antecedente que permita un mayor conocimiento del campo abordado que facilite el acompañamiento y la intervención psicológica en esta población.

\section{REFERENCIAS}

Arias, C. y Polizzi, L. (2011). La relación de pareja. Funciones de apoyo y sexualidad en la vejez. Revista Temática Kairós Gerontología, 14(10), 49-71.

Bagheri-Nesami, M., Rafii, F. y Oskouie, S.F. (2010). Coping strategies of Iranian elderly women: a qualitative study. Educational Gerontology, 36, 573-591. doi: 10.1080/03601270903324438. 
Baltes, P. (1987). Theorical propositions of life-span developmental psychology: on the dynamics between growth and decline. Developmental Psychology, 23(5), 611-626.

Baltes, P.B. y Baltes, M.M. (1990). Psychological perspectives on successful aging: The model of selective optimization with compensation. En P. B. Baltes y M. M. Baltes (Eds.): Successful aging: Perspectives from the behavioral sciences (pp. 1-34). Cambridge, MA: Cambridge University Press.

Baltes, B. y Dickson, M. (2001). Using life-span models in industrial-organization psychology: The theory of selective optimization with compensation. Applied Developmental Science, 5(1), 51-62.

Baltes, P., Lindenberger, U. y Staudinger, U. (1998). Life span theory in developmental psychology. En W. Damon y R. Lerner (Coords.): Handbook of child psychology. Theoretical models of human development (pp. 569-644). New York: Wiley.

Be, D., Whisman, M.A. y Uebelacker, L.A. (2013). Prospective associations between marital adjustment and life satisfaction. Personal Relationships, 20, 728-739. doi: 10.1111/pere.12011.

Bellamy, G., Gott, M., Waterwoth, S., McLean, C. y Kerse, N. (2014). «But I do believe you've got to accept that that's what life's about'»: Older adults living in New Zealand talk about their experiences of loss and bereavement support. Health and Social Care in the Community, 22(1), 96-103. doi: 10.1111/hsc.12069.

Caserta, M., Utz, R., Lund, D, Swenson, K. y De Vries, B. (2014). Coping processes among bereaved spouses. Death Studies, 38, 145-155. doi: 10.1080/07481187.2012.738767.

Consejo Nacional de Población (2014). Proyecciones de la población 2010-2050. México: CONAPO.

Cruz, A., Jara, M. y Rivera, D. (2010). Estrategias de afrontamiento utilizadas por personas adultas mayores con trastornos depresivos. Anales de Gerontología, 6, 31-49.

Damianakis, T. y Marziali, E. (2012). Older adults' response to the loss of a spouse: The function of spirituality in understanding the grieving process. Aging \& Mental Health, 16(1), 57-66. doi: 10.1080/13607863.2011.609531.

Dockendorff, D. (2014). Healthy ways of coping with losses related to the aging process. Educational Gerontology, 40, 363-384. doi: 10.1080/03601277.2013.822203.

Endo, K., Yonemoto, N. y Yamada, M. (2015). Intervention for bereaved parents following a child's death: A systematic review. Palliative Medicine, 29(7), 590-604.

Erikson, E. (1987). Un modo de ver las cosas. Escritos selectos de 1930 a 1980 (Comp. S. Schlein). México: Fondo de Cultura Económica.

Erikson, E. y Erikson, J.M. (1997). The life cycle completed. New York: Norton Co.

Holland, J., Futterman, A., Thompson, L., Moran, C. y Gallagher-Thompson, D. (2013). Difficulties accepting the loss of a spouse: A precursor for intensified grieving among widowed older adults. Death Studies, 37, 126-144. doi: 10.1080/07481187.2011.617489.

Krzemien, D., Urquijo, S. y Monchietti, A. (2008). Adaptación a la crisis vital del envejecimiento: Rol de los estilos de personalidad y de la apreciación cognitiva en adultas mayores. Anales de Psicología, 24(2), 299-311.

Lefrançois, G.R. (2001). El ciclo de la vida (6 ${ }^{\mathrm{a}}$ ed.). México: Ediciones Paraninfo/Thompson Learning.

Li, H., Chi, I. y Xu, L. (2013). Life satisfaction of older Chinese adults living in rural communities. Journal of Cross-Cultural Gerontology, 28(2), 153-165. doi: 10.1007/s10823-013-9189-2.

Liu, W., Liu, H., Wei, D., Sun, J., Yang, J., Meng, J., Wang, L. y Qiu, J. (2015). Abnormal degree centrality of functional hubs associated with negative coping in older Chinese adults who lost their only child. Biological Psychology, 112, 46-55.

Lichtenthal, W.G., Currier, J.M., Neimeyer, R.A. y Keese, N.J. (2010). Sense and significance: a mixed methods examination of meaning making after the loss of one's child. Journal of Clinical Psychology, 66(7), 791-812. doi: 10.1002/jclp.20700.

Montes de O., V. y Macedo de la C., L. (2013). Las redes sociales como determinantes de la salud. En L. M., Gutiérrez y D. Kershenobich (Coords.): Envejecimiento y salud. Una propuesta para un plan de acción (2a ed.) (pp. 121-129). México: Instituto Nacional de Geriatría.

Neimeyer, R. (2007). Aprender de la pérdida. Una guía para afrontar el duelo. Madrid: Paidós.

Oramas, J. (2014). A case study on adjustment to divorce among older Hispanic adults in Miami-Dade, Florida. Journal of Multidisciplinary Research, 6(1), 65-84.

Oviedo S., S., Urdaneta C., E., Parra F., F.M. y Marquina V., M. (2009). Duelo materno por muerte perinatal. Revista Mexicana de Pediatría, 76(5), 215-219.

Rando, T.A. (1985). Bereaved parents: Particular difficulties, unique factors and treatment issues. Social Work, 30(1), 19-23.

Rivera, A. y Montero L., M. (2009). Escala de Estructura de Pérdidas en el Adulto Mayor. En A. L. González-Celis (Comp.): Evaluación en psicogerontología (pp. 31-46). México: El Manual Moderno.

Stefani, D. (2004). Gender and coping in old age. Interdisciplinaria: Revista de Psicología y Ciencias Afines, 22, 109-125.

Tizón, J. (2004). Pérdida, pena, duelo. Vivencias, investigación y asistencia. Madrid: Paidós.

Villar, F. (2005). El enfoque del ciclo vital: hacia un abordaje evolutivo del envejecimiento. En S. Pinazo y M. Sánchez (Dirs.): Gerontología. Actualización, innovación y propuestas (pp.17-43). Madrid: Pearson/Prentice-Hall.

Villar, F., Villamizar D., J. y López C., S. (2005). Los componentes de la experiencia amorosa en la vejez: personas mayores y relaciones de pareja de larga duración. Revista Española de Geriatría y Gerontología, 40(3), 166-177. 


\section{ANEXO}

\section{GUÍA PARA LAS ENTREVISTAS SEMIESTRUCTURADAS}

En el caso de las personas que asistían al club social:

¿Podría hablarme un poco sobre el club social? ¿Hace cuánto tiempo viene? ¿Por qué decidió comenzar a asistir a este club social? ¿Qué actividades son las que más le gustan? ¿Cuándo viene? ¿Cómo se siente?

En el caso de las personas que no asistían al club social:

¿Podría hablarme un poco sobre su vida? ¿Cómo es un día común, desde que se levanta hasta que se acuesta?

\section{INFANCIA}

- ¿Podría platicarme sobre su niñez, de aquellos momentos importantes que le dejaron huella?

- ¿Podría hablarme sobre su lugar de nacimiento y en dónde vivía cuando era niño(a)?

- ¿Y sobre sus padres?

- Lugar que ocupa entre sus hermanos y su relación con ellos.

- Reglas y tradiciones de su familia.

- ¿Cómo era su relación con sus amigos?

- ¿Podría hablarme sobre su escuela y sus compañeros?

- ¿Qué personas fueron las que le brindaron más apoyo cuando era niño(a)?

- ¿Qué problemas tuvo principalmente? ¿Y cómo se enfrentó a ellos?

- ¿Cuáles son los recuerdos más bonitos que tiene de su infancia?

- ¿Y los más desagradables? ¿Hay alguno que le haya dolido más que otro o le haya causado más sufrimiento?

- ¿Qué aprendió de esa experiencia?

- ¿Qué consecuencias tiene eso en estos momentos de su vida?

- ¿Había algo que la preocupara en especial cuando era niño(a)?

- ¿Qué cosas o personas perdió cuando era niño(a)?

- Describa un poco más.

- ¿Qué pensó, sintió o hizo?

- ¿Cómo salió adelante?

- ¿Qué cosas obtuvo cuando era niño(a), es decir, habilidades, experiencias o enseñanzas?

- ¿Dejó de tener algo que era importante para usted?

- ¿Se mudó de casa?

- ¿Tuvo algún cambio de escuela?

- ¿Hubo algún cambio en el estado o en el país que influyera en su vida de alguna manera? (Contexto).

\section{ADOLESCENCIA}

- ¿Qué cambios físicos, emocionales o en su forma de ser experimentó?

- ¿Cómo vivió el paso de la niñez a la adolescencia?

- ¿Cuáles eran sus pasatiempos?

- ¿Podría hablarme sobre sus amigos de esa época?

- ¿Y sus relaciones amorosas?

- ¿Cómo era el ambiente en su escuela?

- ¿Qué maestros tuvo que fueran significativos en su vida?

- ¿Y sus padres? 
- ¿Qué cosas obtuvo cuando era adolescente que antes no tenía?

- ¿Qué fue lo que perdió y dejó de lado?

- ¿Qué problemas importantes tuvo y cómo los resolvió?

- ¿Tenía alguna preocupación cuando era adolescente?

- ¿Cuáles son los recuerdos más bellos que guarda de esta etapa?

- ¿Y los más difíciles o desagradables?

\section{JUVENTUD}

- ¿Cuáles eran sus planes y metas en la vida?

- ¿Cómo fue que eligió su carrera u oficio?

- Respecto al trabajo, ¿cuál fue su primer empleo? ¿Tuvo otros trabajos?

- ¿Cuáles fueron sus logros y fracasos en el área profesional?

En caso de que sea alguien que no cuente con una carrera:

- ¿Cómo se siente con su oficio o trabajo en el hogar? ¿Le gusta hacerlo?

- ¿Qué le gustaba hacer en su tiempo libre?

- ¿Cómo era la relación con sus amigos?

- ¿Podría hablarme sobre las parejas que tuvo en ese tiempo?

- ¿Cómo fue que conoció a su esposo(a)?

- ¿Podría hablarme más sobre su matrimonio?

- ¿Qué cosas obtuvo cuando era joven que antes no tenía?

- ¿Qué fue lo que perdió?

- ¿Había algo que la preocupara?, ¿qué era?

- ¿Cuáles son los recuerdos más bellos que guarda de esta etapa?

- ¿Y los más difíciles o desagradables?

\section{ADULTEZ}

- ¿Podría hablarme sobre los eventos o acontecimientos que mayor impacto tuvieron en su vida?

- ¿Qué podría decirme sobre su familia?

- ¿Qué pasó respecto a su trabajo?

- ¿Cuáles considera que fueron sus mayores logros en general?

- ¿Qué cosas o personas importantes para usted perdió en esa época? ¿Qué fue lo que perdió o ha perdido?

- ¿Qué problemas o situaciones difíciles se le presentaron en esta época?

- ¿Cómo salió adelante?

- ¿Con quién contaba cuando tenía un problema o situación difícil?

- ¿Qué cosas obtuvo como adulto(a) que antes no tenía?

- ¿Cuáles son los recuerdos más bellos que guarda de esta etapa?

- ¿Y los más difíciles o desagradables?

\section{ADULTEZ MAYOR}

- ¿A qué cambios se ha enfrentado en este momento de la vida en que se encuentra?

- En esta etapa de su vida, ¿qué cree que puede perder?

- ¿Y qué cosas cree que pueden ganarse?

- Desde su experiencia personal, ¿qué cosas que no tenía antes posee ahora?

- ¿Y cuáles ha perdido?

- En estos momentos de su vida, ¿ha perdido algo o a alguien?

- ¿Qué le ayudó a salir adelante, a adaptarse?

- ¿Qué suele hacer cuando se enfrenta a un problema o situación difícil? 
- ¿Podría hablarme de las principales personas que lo apoyan y están ahí para usted cuando lo necesita?

- (En caso de que hable de enfermedad) Ahora que enfermó ¿qué pensó, que sintió y qué hizo?

- ¿Cuáles son los recuerdos más bellos que guarda de esta etapa?

- ¿Y los recuerdos más difíciles o desagradables?

- ¿Hay algo que le preocupe actualmente?

- Si tuviera que enlistar los acontecimientos y logros más importantes de su vida, ¿cuáles serían?

- ¿Qué cosas ha aprendido de su vida? 